\title{
Celebrating Forty Years of Clinical Social Work
}

\author{
Carol Tosone
}

Published online: 25 January 2013

(c) Springer Science+Business Media New York 2013

This March 2013 issue marks the 40th anniversary of the Clinical Social Work Journal (CSWJ). The brainchild of Mary Gottesfeld, a clinical social worker at the Jewish Board of Family and Children's Services in New York City, the CSWJ was established in 1973 as a publication forum for the National Federation of Societies for Clinical Social Work (NFSCSW). Mary served as the CSWJ's Founding Editor until 1981, and during her tenure published numerous articles on ego psychology and its application, as well as the impact of deinstitutionalization on treatment, the efficacy of psychotherapy, and the emergence of behavioral therapies.

As the NFSCSW grew in membership, the CSWJ enjoyed a substantial increase in its readership. Mary's untimely illness necessitated her turning the reigns of the editorship to Jean Sanville, a nationally known psychoanalyst and scholar who presided skillfully over the CSWJ and its editorial board for 15 years. Jean viewed psychoanalytic treatment and clinical social work as kindred approaches, and the articles published during her tenure reflected this position. No longer was ego psychology the dominant paradigm. Object relations theory, especially applied to the understanding of borderline personality disorder and countertransference reactions, were major themes for nearly a decade.

Social issues and diagnostic entities such as AIDS, sexual abuse, and substance abuse came to the fore in the CSWJ during the 1990s. Managed care intruded into the clinical hour, as did concerns regarding licensure, professionalism, and the relevance of long-term psychoanalytic

C. Tosone $(\bowtie)$

New York University Silver School of Social Work, New York, NY, USA

e-mail: ct2@nyu.edu treatment in a short-term, evidence-based practice (EBP) world. During Carolyn Saari's ten-year tenure as Editor-inChief, she and the editorial board served as able stewards of clinical social work. Keeping loyal to the original intent of the journal, these issues were addressed and illustrated through a largely psychodynamic lens.

The dissolving of the NFSCSW coincided with the change of Editors. Carolyn's daunting charge to me in 2007 was to find creative ways to keep the CSWJ current with professional and world developments without abandoning its core psychoanalytic social work mission. While a crucial component of contemporary social work practice, the psychodynamic orientation has had to share the stage with EBPs and related manualized treatments, as well as shortterm integrative treatments for specialized populations. Proponents of psychoanalytic social work have also had to combat the general perception that psychodynamic treatment is anachronistic and suitable only for the worried well.

Coupled with extrinsic factors as varied as HIPAA regulations and global terrorism, the $C S W J$ has responded by broadening the definition of clinical social work to encompass all contemporary models and treatment approaches that benefit clients, especially those populations most at-risk. Special issues on empirically supported attachment-based therapies for adults and children, emblematic of the current psychoanalytic zeitgeist, have been followed by issues devoted to integrative treatment, EBP research, international trauma, family therapy, and agency-based practice with vulnerable populations to name a few. It is therefore fitting that this March 2013 special issue is devoted to culture and creativity in contemporary psychoanalytic thought. A comparison to the first issue of the CSWJ in March 1973, this issue attests to the evolution of the psychoanalytic approach and its rightful place along side EBP in the clinical social work armamentarium. 
No longer affiliated with a particular professional organization, the current incarnation of the CSWJ is dedicated to enhancing the practice wisdom of clinicians, whether in agency-based or private practice settings. To do so in these demanding times requires providing clinicians, as well as social work educators and their students, with articles that articulate relevant practice research findings, promising diagnostically-based practice models, and the application of widely accepted models to diverse populations. Thanks to the dedication of Carol Ganzer, Managing
Editor, and the editorial board, the content of the CSWJ maintains practical applicability for front line clinicians. Geoffrey Golia, Social Media Manager, increases awareness of and access to the $C S W J$ through a sustained presence on Twitter and Facebook.

My fondest wish for the CSWJ readership is to be enjoying the centennial issue of the journal in 2073, and to find its content fresh, thought provoking, germane, and especially au courant. Until then, happy reading! 